\title{
APPLICATION OF NANOFILTERS FOR VENTILATION
}

\author{
ALEŠ RUBINA, OLGA RUBINOVÁ, PETR BLASINSKI* \\ Institute of Building Services, Brno University of Technology, Faculty of Civil Engineering, \\ Veveří 331/95, 60200 Brno, Czech Republic \\ *E-mail: petr.blasinski@vut.cz
}

\begin{abstract}
Nanotechnology is a perspective manufacturing technology, and in the technical fields, it deals with the production, development and utilization of technologies and materials with dimensions in nanometre sizes $(1-100 \mathrm{~nm})$. Nanofilters used in the article for filtration purposes consist from a nanolayer which is applied to a coarse textile backing layer, and they are inserted into the frames as conventional textile filters. The most commonly used materials are PP and PE polymers, as well as carbon, glass and metal filters. With the fabrication of nanotechnology-based filter, it is very important to choose materials, polymers with specific properties, which can be used for filtration function of the product itself. The results given in the main article compare the nanofilters with the main representatives of existing filter products currently available on the market. There is a problem with high pressure loss of the nanomaterial, and when we compare them with traditional filters, it is difficult to use them in technical practice, even if there exists the possibility for us to define the material and the thickness of the layer which are adapted to the application-specific application conditions.
\end{abstract}

Keywords: ventilation, filtration, separability measurements, air cleanness, nanofibers

\section{Introduction}

Ensuring air filtration for low-dust ventilation in the air supply is the primary function of even the simplest ventilation systems. In accordance with the legally binding and normative regulations, it is necessary to filter not only the freshly supplied, but also the discharged degraded air, and provide it with the appropriate degree of filtration according to the type and size of pollutants. The subject of this article is not the draft of the concept or of the level of filtration itself, but the introduction of a new material which begins to appear in the offer of selected Czech and foreign producers. These filters are made from nanofibered structures. The paper presents an experimental prototype filter which is an alternative to contemporary microfiber air filters for F9 filtration levels. The presented measurement results are carried out by the scientific workers of the Faculty of Civil Engineering, Brno University of Technology, the Institute of Building Services.

Nanotechnology is a promising manufacturing technology, and in fields of technology, it deals with the production, development and utilization of nano- materials $(1-100 \mathrm{~nm})$. In various sectors of the construction industry, nanotechnology has found its permanent place, improving the properties of commonly used products.

In case of the use of nanofiber materials for the needs of air filtration in the air-conditioning industry, the products are only marginally to be had on the market. The text of this article tries to elucidate the present state of the issue, its basic advantages and disadvantages.

\section{Materials and calculation methods}

Production of nanofiber filters consists of applying nanofibers to a coarse textile backing layer and then they are processed as a classic textile liner into the frames of the filters.

The technology used for the production of nanofilters is called electrospinning or electrostatic spinning. It is possible to process almost all types of polymers, mostly in form of liquid. To produce the fibers, it is necessary for the polymer to have a sufficiently low viscosity. The diagram of electrostatic spinning is shown in Fig. 1.

This is an open-access article distributed under the terms of the Creative Commons Attribution-NonCommercial 4.0 International License (https://creativecommons.org/licenses/by-nc/4.0/), which permits unrestricted use, distribution, and reproduction in any medium for non-commercial purposes, provided the original author and source are credited, a link to the CC License is provided, and changes - if any - are indicated. 


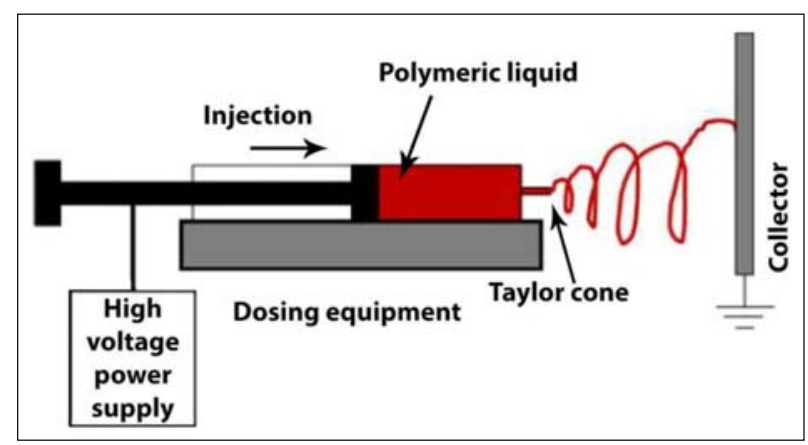

Fig. 1. Scheme of electrostatic spinning (principle) [1]

The polymeric liquid is directly connected with the high voltage power supply electrode, which is then spun by a spinneret. Due to the high voltage, sometimes up to $50 \mathrm{kV}$, the Taylor cone is formed, namely between the tip of the capillary and the grounded collector. It produces so-called submicron fibers, which solidify by evaporating the solvent and form a fibrous layer. This is settled on the collector surface due to the charged field. As collector, a textile material is commonly used [2].

The structure of the nanofiber layer is formed by the classic spinning, and the result is presented in Fig. 2.

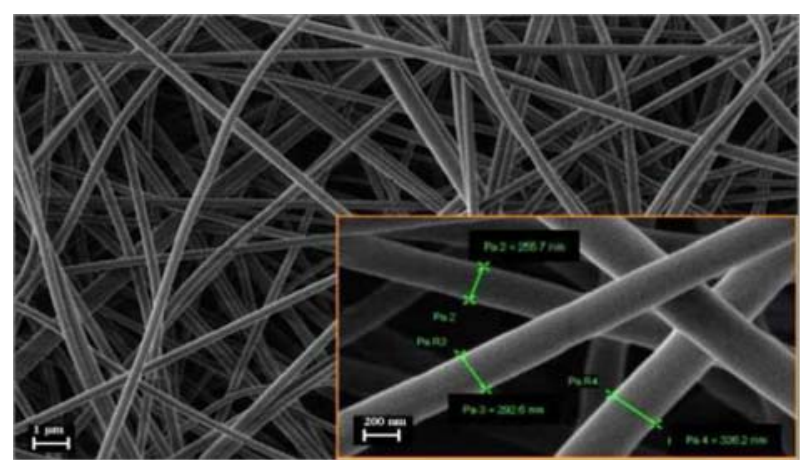

Fig. 2. Photographs of applied nanofibers [1]
By electrostatic spinning, a wide range of polymers can be prepared. The most commonly used material for the production of nanofibers is a solution of PP and PE polymers, as well as carbon, glass and metal fibers. The production of nanofibers can be divided into melts, polymer or aqueous liquids. Each of these types of production has its advantages and disadvantages. For the production of textile materials with the help of nanotechnology, it is very important to choose materials (polymers) with specific properties which may also influence the function of the product itself.

Figure 3 (left) shows the structure of the filtration stage degree G3 (scale 1:50), illustrated by an electron microscope TESCAN VEGA 3, and in Fig. 3 (right), there is a four-nanofiber filter (scale 1:150). Although the scale for nanofibrous material is three times larger, the material is significantly thicker and more regularly stratified.

The incorporation of nanofilters into the normal distribution used in practice is quite problematic, because the structure of the material is different.

There is no analogy between the thickness or square weight of the produced layer and the functional properties of the material.

Differences were also found between laboratory-measured values on small samples elastically strained in a tight frame and the final product stacked into a shape and design usable in ventilating equipment.

Therefore, the filter design, classification into the filtration degree, and the resulting pressure loss, significantly affecting the performance of the fan and its operation costs, are essential.

Filter distribution according to the structural design:

$$
\begin{aligned}
& \text { - Frame-Z-line } \\
& \text { - Pocket } \\
& \text { - Complat }
\end{aligned}
$$

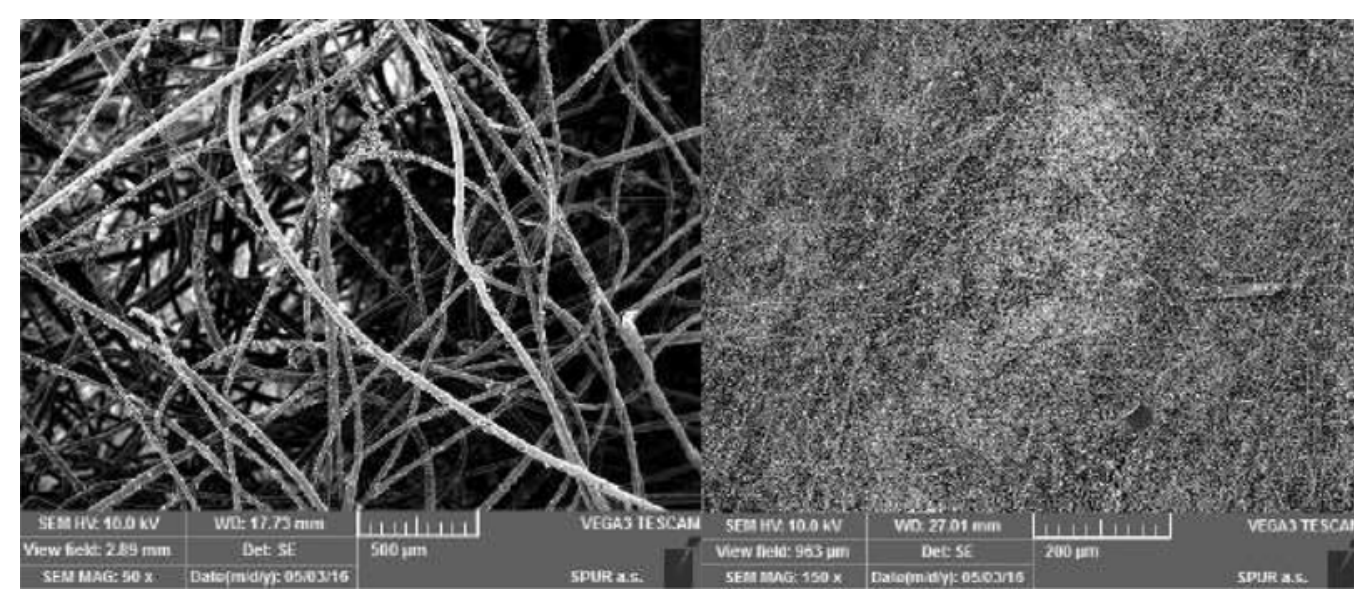

Fig. 3. Standard filter G3 $195 \times 195 \mathrm{~mm}$ (left), nanofilter $195 \times 195 \mathrm{~mm}$ (right) 


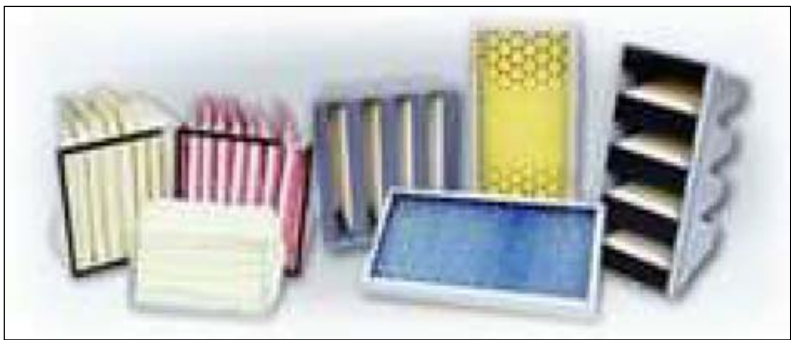

Fig. 4. Example of different types of mechanical filters used in ventilation

Filter distribution according to the frame:

- Metal frame

- Plastic frame

- Tempered paper frame

Filter distribution according to the fabric connections:

- Sewn by textile thread

- Ultrasonic welding

While the classic sewn filter has a long history, ultrasonically welded filters are relatively new in the market. Ultrasonic welding takes place using friction heat generated by high-frequency mechanical vibrations of about $15-75 \mathrm{kHz}$. High-frequency of electric energy is converted to mechanical one. After that, a specially shaped sonotrode is used to bond the material by which friction heat is produced. The result of this is local melting of the bonded material. When the temperature at the interface of bonded materials reaches the melting point, the material melts and flows. Using the controlled sonotrode pressure at the interface of interconnected parts, the material cools and a molecular weld is formed [3].

From the design point of view, it is possible to produce nanofilters in any design. The test sample measured in the laboratory was made as a pocket design filter in a plastic frame. Both types ultrasonically welded and sewn joints were tested.

Integration into the filtration class. Atmospheric air filters for separation of particles in normal ventilation are tested and sorted according to the European Standard ČSN EN 779.

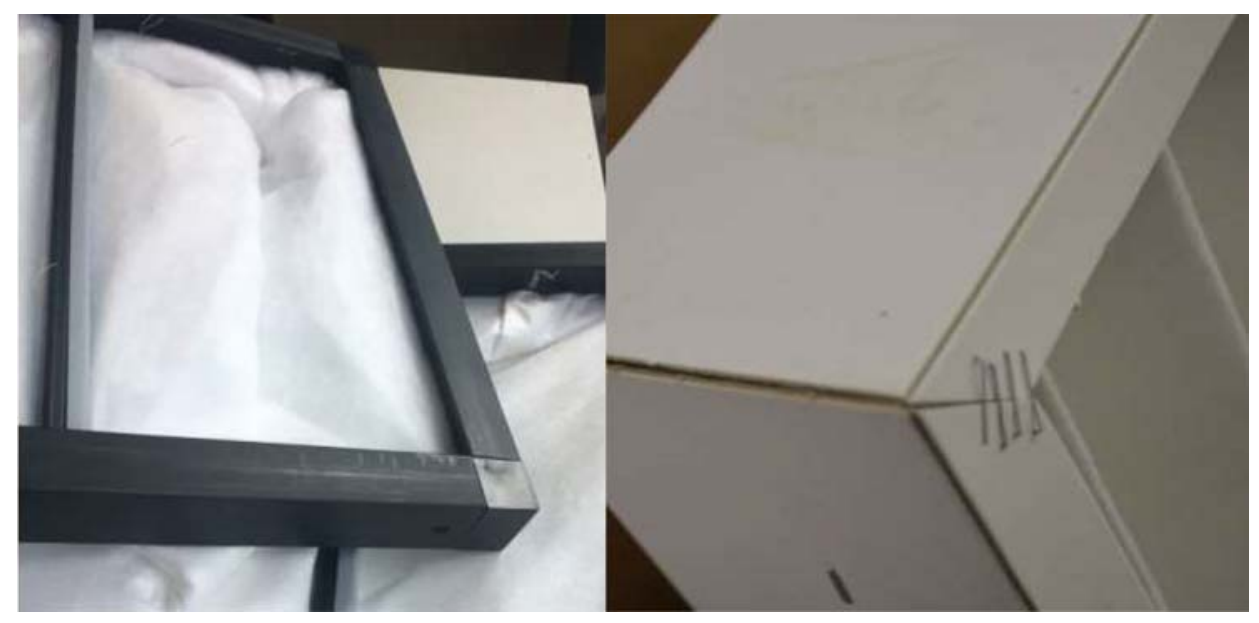

Fig. 5. Sample of plastic (left) and paper frames (right)

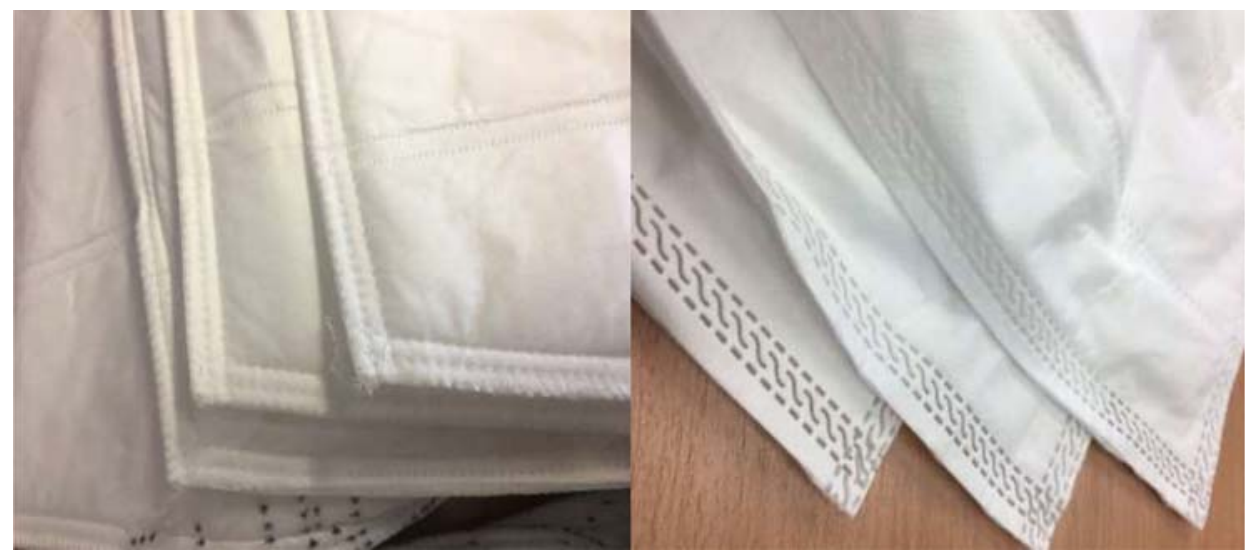

Fig. 6. Example of a pocket filter with sewn (left) and ultrasonic welded (right) filters 
The filters are as follows:

- $G$ for coarse dust - effective for particles $>10 \mu \mathrm{m}$ (G1, G2, G3, G4)

- $\mathrm{F}$ for fine dust - effective for particles $>1 \mu \mathrm{m}$ (F5, F6, F7, F8, F9).

High efficiency filters that are tested and sorted according to EN 1822:

- $\mathrm{H}$ for microparticles - effective for particles $>0.01 \mu \mathrm{m}(\mathrm{H} 10, \mathrm{H} 11, \mathrm{H} 12, \mathrm{H} 13, \mathrm{H} 14)$

- U for microparticles (U15, U16, U17).

The tested prototype of the functional sample of the filter is outlined as a fine-grain filter of F9 type.

Arrestance $A_{j}$ is measure of the ability of filter to remove a standard test dust from the air passing through it under given operating conditions expressed as a weight percentage:

$$
A_{j}=\left[1-\left(m_{j} / M_{j}\right)\right] \cdot 100[\%],
$$

where $m_{j}$ is the weight of the dust penetrating the filter (the weight gain of the end filter $\Delta m_{f f}$ and the dust weight in the channel behind the filter $m_{d}$ ) at the dust loading phase $j, M_{j}$ is the mass of the dust supplied (dust dose $\Delta m$ ) during the loading phase $j$.

The mean separation $A_{m}$ is the average of five individual isolation values of $A_{j}$ :

$$
A_{m}=(1 / M)\left[M_{1} A_{1}+M_{2} A_{2}+\ldots+M_{n} A_{n}\right][\%],
$$

where $M=M_{1}+M_{2}+\ldots+M_{n}$ is the total mass of the dust supplied, $M_{1}, M_{2}, \ldots, M_{n}$ are the masses of dust gradually fed into the track until the final pressure drop $\Delta p_{1}, \Delta p_{2}, \ldots, \Delta p_{n}$ has been reached.

Pressure loss of the filter. The filter pressure loss is the resistance that occurs when air flows through the filter, and by the filter closure due to it. In their projection bases, manufacturers present the initial pressure loss of the filter, the mean pressure drop, and the final pressure loss of the filter at its full closure. In practice, nominal design airflows should be given for the mean pressure drop of the filter. In general, it can be characterized that the pressure drop of the filter depends on the square of the air flow velocity and on the hydraulic resistance of the insulated fiber $F_{\gamma}$. The fiber resistance is then equal to the pressure difference in the given layer.

The form of the test track of measuring the filters is shown in Fig. 7. Filter pressure loss is the pressure difference between points $p_{s 3}$ and $p_{s 4}$.

\section{Results}

Arrestance. The measured arrestance of the nanofiber filter is shown in Figs 8, 9 and 10. The filter sample of a four-pocket design in a plastic frame is concerned. The nanofiber filter is joined both by sewing joint and ultrasonic welding. The sample was placed in the test track so that the pockets were in vertical position and the surface of the filter was not degraded due to its own weight.

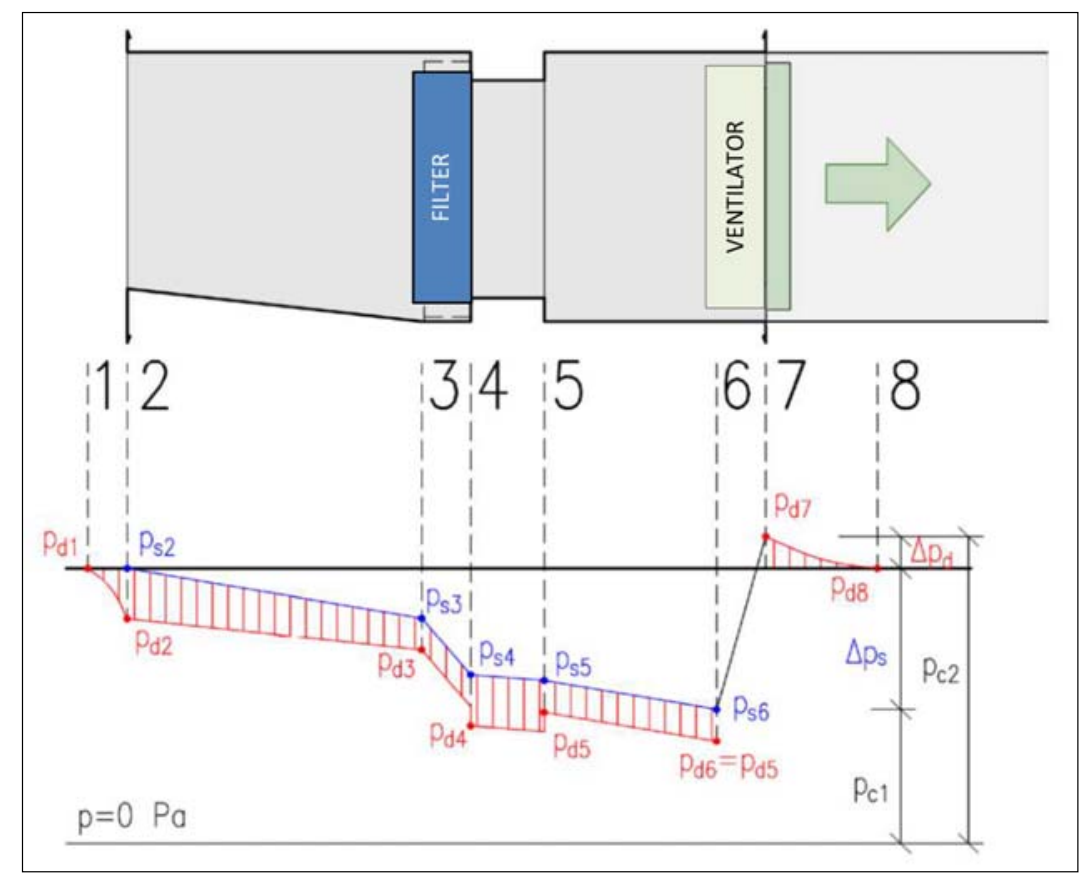

Fig. 7. The shape of the test track 


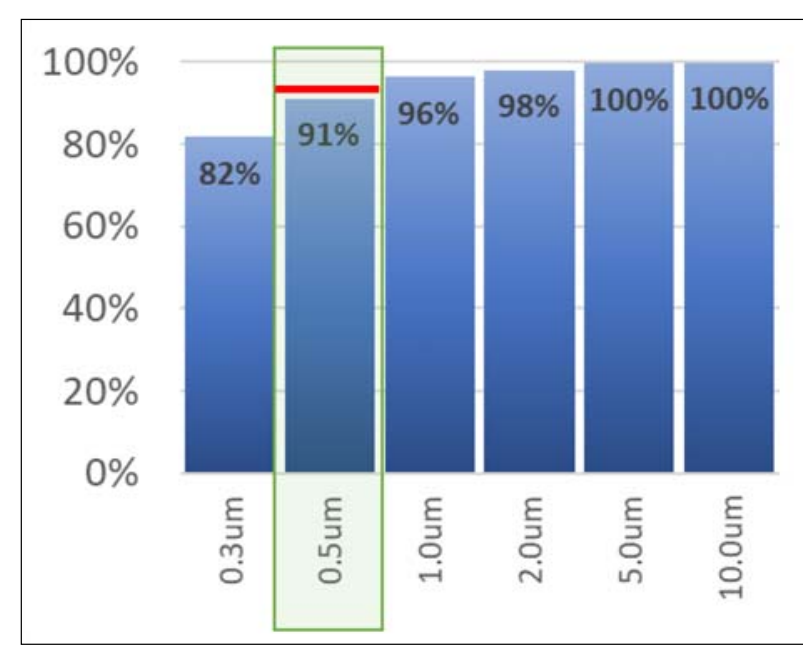

Fig. 8. Measurement of the arrestance of four-pocket filters made from nanofibers with an ultrasonic welded seamed joint

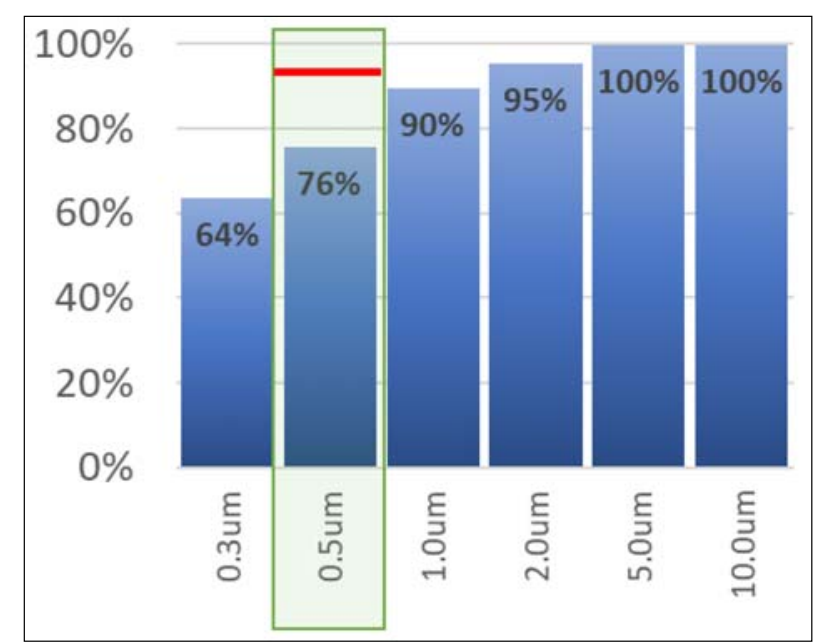

Fig. 10. Measurement of the separability of a commonly sold four-pocket filter - the F9 filter degree

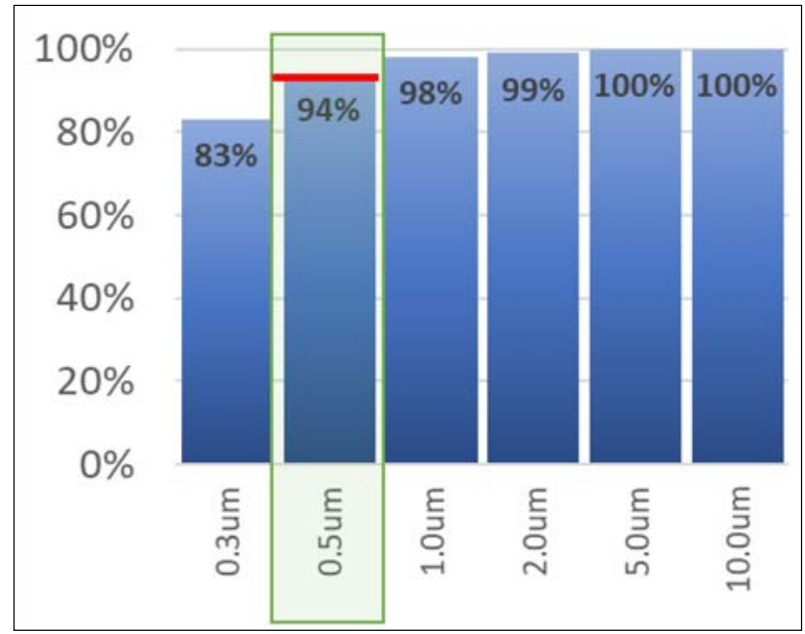

Fig. 9. Measurement of the arrestance filters from nanofibers with ultrasonic welded joint

Pressure loss of the filter. See Fig 11.

\section{Discussion}

Arrestance. Between the joints in the nanofiber filters for a $0.5 \mu \mathrm{m}$ fraction, there is $4 \%$ degradation, with improvements occurring in all fractions. The classical four-pocket filter of polyester fibers (F9 filtration degree) presents only $76 \%$ of segregation.

Pressure loss of the filter. It can be seen from the graph in Fig. 10 that, at the air flows of $400 \mathrm{~m}^{3} / \mathrm{h}$ and above, the demand for nanofilters with sewn connection for the transport pressure of a given amount of air is by

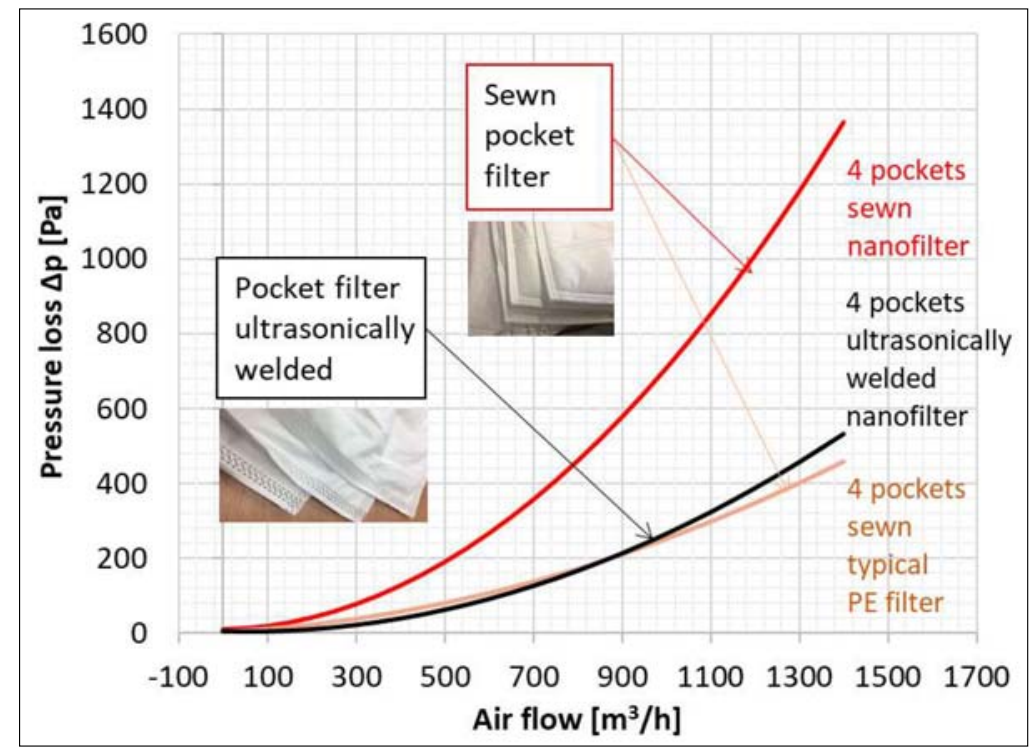

Fig. 11. Measurement of pressure loss of four-pocket filters of nanofibers and a classical four-pocket filter of polyester fibers of F9 filtration degree 
about $60 \%$ higher than for a standard F9 polyester fiber textile filter.

Nevertheless, the pressure loss is almost comparable in the ultrasonically welded filter, in comparison with the standard textile filter. It varies with flow rates above $950 \mathrm{~m}^{3} / \mathrm{h}$, if the result does not rise by more than $10 \%$ of the measured value.

For the results of the pressure loss measurement on the same material, the effect of connection is very noticeable.

\section{Conclusion}

In the Czech Republic, the technology of production of nanofibrous textiles is very advanced, and it is possible for us to define the material and the thickness of the applied layer in compliance with the frames of manufacturing industry.

The advantage of smaller filaments can be a larger range of density selectivity of the nanotextile filter material, and thus greater selectivity of particle separability by the filter material. The standard EN 799 and EN 1822 require fulfilling a certain arrestance of air particles (fine dust and microparticles) to be achieved for the filter classification into a degree of filtration. Due to the high-pressure loss of material compared to conventional filter inserts, nanofilters have been unusable in technical practice for a long time. From the preliminary results of measurements on prototypes of basic nanofilters, it is clear that, by improving used technology, the pressure loss can be similar to the commonly used products, and the result of the particle separation is significantly better.

\section{Acknowledgements}

The article was created with the support of the specific research project No. FAST-S-17-4054. Materials for testing were provided by ASIO comp. s.r.o. and SPUR a.s.

\section{References}

[1] Nanopharma - Technology. [online]. Copyright (C) 2015 nanopharma [online]. Available from: http://www. nanopharma.cz/en/products-and-technology/technology

[2] Kučerová L. (2017), Detection of Deformation and Voltage by Polymer Nanocomposite with Integrated Carbon Nanotube Layer. Bachelor Thesis, Tomas Bata University in Zlín, Faculty of Technology, [online].

[3] Ultrasonic welding line, Temex. Temex [online]. Available from: http://www.temex.cz/katalog/produkty-a-sluzby/ reference/331/ultrazvukova-svarovaci-linka.html

[4] ČSN EN 779 (12 5001) (2012), Atmospheric air filters for particle separation for general ventilation - Determination of filter parameters. Prague: Office for Standards, Metrology and Testing.

[5] ČSN EN 1822-1 (125002) (2010), Highly efficient air filters (HEPA and ULPA) - Part 1: Classification, verification of properties, labeling. Prague: Office for Standardization, Metrology and Testing. 